\title{
Pharmacological management of treatment-resistant depression
}

\author{
P. J. Cowen
}

The management of patients with depression who have failed to respond to antidepressant medication is a common problem in general and old age psychiatry. It has been estimated that about $20-30 \%$ of patients with major depression fail to respond to treatment with a single antidepressant drug given in adequate dosage for an appropriate period of time. At the current time there are many possible ways to pursue pharmacological treatment, but few controlled trials to help us choose between the various options. In addition there are few clinical predictors to help match patients to an appropriate treatment.

\section{Definition}

The definition of treatment-resistant depression is somewhat arbitrary, but in pharmacological terms there is the presumption that the depressive syndrome has not responded to an adequate trial (in terms of dose and duration) of at least one effective medication. It may be useful to identify different stages of resistant depression depending on the nature of treatments that have been unsuccessfully deployed (Box 1; Thase \& Rush, 1997). Some patients may present with chronic untreated depression that has failed to remit spontaneously. Such patients can respond well to pharmacotherapy, although there is disagreement about whether remission rates are lower than in depressed patients with more acute presentation (Kupfer \& Frank, 1996).

\section{Assessment}

Patients with depression are often now referred to psychiatrists after some considerable period of illness and after a number of different drug treatments have been tried. It is important to confirm the diagnosis of major depression and exclude possible general medical disorders. Some medical treatments may themselves precipitate or perpetuate depression; for example, recent epidemiological studies have drawn attention to excess rates of depression and suicide in patients taking calcium channel antagonists (Lindberg et al, 1998). Psychiatric comorbidity, for example substance misuse, can also worsen the outcome of depressive disorder.

The assessment should attempt to establish how long the depression has been present, its nature and course and the treatments that have been used. Often, patients will have improved to some extent and this may influence whether or not major changes in management are needed. It is helpful to try to
Box 1. Stages of treatment resistance in depression (adapted from Thase \& Rush, 1997)
Stage 1 Failure of at least one adequate trial of one major class of antidepressant
Stage 2 Stage 1 plus failure to respond to adequate trial of antidepressant from a different class
Stage 3 Stage 2 plus failure to respond to lithium augmentation
Stage 4 Stage 3 plus failure to respond to monoamine oxidase inhibitor
Stage 5 Stage 4 plus failure to respond to electroconvulsive therapy

Philip Cowen has studied basic neuropharmacology and its application to clinical psychopharmacology. Since 1983 he has been an MRC Clinical Scientist and Honorary Consultant Psychiatrist at the University Department of Psychiatry, Oxford (Warneford Hospital, Oxford OX3 7JX). His main interests are the biochemistry and treatment of mood disorders. 
establish how far psychosocial and personality factors may have played a role in the onset and maintenance of the depression. For this purpose it is usually necessary to interview an informant. Although knowledge of these factors does not necessarily affect selection of drug treatment, it can influence prognosis.

\section{General measures}

All patients need psychotherapy, in the general sense of supportive listening, reassurance and education. Often, patients with chronic depression are understandably demoralised, pessimistic and despairing. It is usually helpful to outline the treatment plan as a collaborative exercise in which the clinician uses their skill and experience to select potentially useful therapeutic options while the patient's task is to stay in treatment and engage in normal day-to-day activities as far as possible. Cognitive techniques can be useful in helping patients to focus on achievements and identify and deal with negative thinking patterns.

\section{Medication}

\section{Dose-response issues}

Many patients taking tricyclic antidepressants (TCAs) are treated with relatively low doses and may improve when the dose is increased to 150 $200 \mathrm{mg}$ daily. In individual subjects, where tolerance is good, additional benefit may be obtained by increasing the dose further, up to $300 \mathrm{mg}$ of imipramine or its equivalent. Plasma monitoring of tricyclic levels may be helpful at higher doses to guard against toxicity, particularly if there is a possibility of pharmacokinetic interactions (Preskorn, 1993). In addition, when using high doses it may be prudent to monitor patients who have any previous history of cardiac disorder or who are taking other medication that might impair cardiac conduction.

Venlafaxine resembles TCAs, in that higher doses have greater efficacy, but selective serotonin reuptake inhibitors (SSRIs) are said to have relatively flat dose-response curves. Despite this, if tolerance permits, increasing the dose of an SSRI can produce symptomatic improvement, particularly in patients who have shown a partial response (Fava et al, 1994).

\section{Switching antidepressants}

If a patient does not respond to one antidepressant drug, it is comparatively simple to try a different preparation. Most of the published studies of this treat patients in an open, sequential way with a different class of antidepressant; clearly this cannot control for the placebo effect or the possibility of spontaneous remission. There is reasonable evidence that switching to another class of antidepressant can produce benefit in about $50 \%$ of patients unresponsive to an initial medication.

If a patient has not responded to one kind of antidepressant, it would seem sensible to switch to an antidepressant with different pharmacological properties - this is my practice. However, it must be acknowledged that open studies have shown equally good response rates when patients who failed to respond to one SSRI were switched to another (Thase \& Rush, 1997).

In the broad range of patients with depression, currently marketed antidepressants have roughly equal efficacy. However, there is evidence from meta-analyses that drugs that produce potent potentiation of both serotonin and noradrenaline (amitriptyline, clomipramine and venlafaxine) may be more effective than SSRIs in patients with severe depressive symptoms (Anderson, 1997). This suggests that one of the former drugs should be tried at some point in patients unresponsive to initial medication.

\section{Monoamine oxidase inhibitors}

The use of non-selective irreversible monoamine oxidase inhibitors (MAOIs) in patients resistant to TCAs and other antidepressants has some support from controlled trials. For example, Nolen et al (1988) studied 21 patients who had failed to respond to treatment with imipramine, fluvoxamine or oxprotiline. The subjects were randomly allocated to double-blind treatment with nomifensine, a dopamine and noradrenaline reuptake inhibitor (later withdrawn because of rare autoimmune reactions) or the MAOI tranylcypromine. Of 11 patients receiving tranylcypromine, five responded with a $50 \%$ decrease in their score on the Hamilton Rating Scale for Depression (HAM-D; Hamilton, 1967). In a subsequent cross-over, five of eight nonresponders to nomifensine responded to tranylcypromine. Eight of the 10 patients who responded to tranylcypromine, maintained their response for at least six months.

There is also evidence that patients with certain clinical features may have a preferential response to MAOIs. For example, patients with symptoms of 
atypical depression (see Box 2) have a significantly higher response rate to phenelzine (about $70 \%$ ) than imipramine (about 45\%; Quitkin et al, 1989).

Although MAOIs are undoubtedly useful drugs in treatment-resistant depression, their liability to produce dietary and drug interactions makes their use unlikely until most other options have been exhausted. The reversible type-A MAOI, moclobemide, is relatively free from tyramine interactions at standard doses and is better tolerated than conventional MAOIs. However, there is only limited evidence for its usefulness in resistant depression.

Stablet al (1995) compared moclobemide ( $450 \mathrm{mg}$ daily) with a combination of moclobemide and thioridazine in the treatment of 78 depressed in-patients who had failed to respond to at least two previous trials of antidepressants. Overall, about $75 \%$ of the patients showed a useful clinical response ( $50 \%$ decrease in HAM-D score) with no difference between the two treatment regimes. Thus, moclobemide can be worth trying in treatmentresistant patients but if it proves ineffective, a switch to a conventional MAOI may still be warranted (Cowen, 1998).

A key issue for successful treatment with MAOIs is the use of adequate doses. For example, some patients may require up to $90 \mathrm{mg}$ a day of phenelzine or $60 \mathrm{mg}$ a day of tranylcypromine. With higher doses it is wise to monitor blood pressure for the development of postural hypotension.

\section{Augmentation strategies}

A problem in switching antidepressant preparations is that withdrawal of the first compound may not be straightforward. Patients may have gained some limited benefit from the treatment, for example, in terms of improved sleep or reduced tension, and this will be lost. In addition, if the first medication is stopped quickly, withdrawal symptoms may result. Alternatively, gradual tapering of the dose makes the change-over in medication rather protracted, which may not be easily tolerated by a despairing patient with depression. For this reason, in patients

Box 2. Clinical predictors of response to conventional MAOIs

(a) Atypical depression - mood reactivity overeating, oversleeping, overwhelming fatigue, rejection sensitivity

(b) Bipolar depression with anergia and hypersomnia unresponsive to first-line medication, it may be more appropriate to add a second compound to the primary antidepressant, in the hope of producing an additive or even synergistic effect. The major disadvantage of this procedure is that that the risk of adverse effects through drug interaction is increased.

\section{Antipsychotic drugs}

Naturalistic studies have shown that patients with depressive psychosis have low response rates to treatment with TCAs alone but may respond well when antipsychotic drugs are combined with a TCA. In a double-blind, random allocation study of 51 inpatients with depressive psychosis, Spiker et al (1985) found that the response rate (final HAM-D $<7)$ to amitriptyline alone was $41 \%$, while that to perphenazine alone was $19 \%$. However, patients receiving combined treatment with these drugs had a significantly higher response rate $(78 \%)$ than either of the other two groups.

Most of the reports of the drug-treatment of depressive psychosis have involved TCAs. However, in an open study Rothschild et al (1993) found that the combination of fluoxetine (up to $40 \mathrm{mg}$ daily) and perphenazine (up to $35 \mathrm{mg}$ daily) produced a $73 \%$ response rate (reduction in HAM-D rating of at least $50 \%$ ) in 30 patients with DSM-III-R (American Psychiatric Association, 1987) depressive psychosis.

It therefore appears that a combination of SSRIs and antipsychotic drugs may be effective in depressive psychosis. However, in the above study 14 patients developed tremor and two experienced akathisia. SSRIs can cause extrapyramidal movement disorders and may potentiate the extrapyramidal effects of antipsychotic drugs through both pharmacodynamic and pharmacokinetic interactions (Young \& Cowen, 1994).

There is interest in the role of new antipyschotic agents such as risperidone and clozapine in depressive psychosis, and a number of positive case reports and series have appeared. However, a controlled trial found that risperidone monotherapy was significantly less effective than a combination of amitriptyline and haloperidol in patients with psychotic depression (MullerSiecheneder et al, 1998).

\section{Lithium}

Lithium given alone has modest antidepressant properties in patients with bipolar disorder, but other depressed patients show little response. There is now, however, good evidence from uncontrolled and controlled trials that lithium added to ineffective antidepressant treatment can produce 
useful clinical improvement in patients with major depression. Whether this effect of lithium is a true potentiation (augmentation) of the primary antidepressant compound or simply represents an additive antidepressant effect of its own is debatable.

Uncontrolled trials have reported that lithium addition is followed by a rapid onset of antidepressant effect (within 48 hours) in a high proportion of subjects $(60-70 \%)$. Double-blind, placebo controlled trials confirm that lithium is effective, but shows a more gradual onset of action over $2-3$ weeks in about $40-50 \%$ of patients with depression (Cowen, 1998).

Lithium appears to be effective in improving antidepressant response when added to different kinds of primary antidepressant treatment, including TCAs, SSRIs and MAOIs (Johnson, 1991). Caution is needed when using lithium together with SSRIs because both treatments combine to potentiate brain 5-HT function leading to a risk of 5-HT neurotoxicity. This combination, however, does not seem to have greater therapeutic efficacy than other lithium-antidepressant combinations, despite the marked increase in brain 5-HT function that it produces (Katona et al, 1995). On the basis of open studies it has been suggested that the combination of lithium with an MAOI may be particularly helpful in treatment-resistant depression (Price et al, 1985).

The plasma level of lithium required to produce an antidepressant effect in treatment-resistant patients has not been clearly established, but levels of $0.5-0.8 \mathrm{mmol} / \mathrm{l}$ are usually adequate. It is usually best to initiate lithium treatment at a low dose, for example $200-400 \mathrm{mg}$ daily, particularly where patients are taking serotonergic antidepressants such as SSRIs and MAOIs.

\section{Triiodothyronine}

Several open studies have indicated that the addition of triiodothyronine to ineffective TCA treatment can bring about a good clinical response, and this has been supported in three out of four controlled studies. In the most recent controlled investigation, Joffe et al (1993) studied 50 out-patients with unipolar, non-psychotic major depression. They had failed to respond to five weeks' treatment with a TCA (daily dose $2.5 \mathrm{mg} / \mathrm{kg}$ ) after which they were randomly allocated to double-blind addition of lithium carbonate, triiodothyronine ( $37.5 \mathrm{mg} /$ day) or placebo for two weeks. At the end of treatment 10 of 17 patients treated with triiodothyronine had responded $(50 \%$ reduction in HAM-D with final HAM-D score $<10$ ). A similar response rate (nine of 17 patients) was noted in patients receiving lithium, while only three of 16 subjects responded to placebo.

The data suggest that addition of triiodothyronine is a useful means of augmenting TCA treatment in patients with depression. In the UK, triiodothyronine is available as a $20 \mathrm{mg}$ tablet (equivalent to about $100 \mathrm{mg}$ thyroxine). When added to TCAs this dose is usually well tolerated; however, caution should be used in patients with cardiovascular disease. If this dose is unsuccessful but tolerance is good there is the option of increasing to $40 \mathrm{mg}$. At the higher dose symptoms of tachycardia, sweating, hot flushes and anxiety may be experienced. There are at present no comparative data to show that triiodothyronine can augment the action of other classes of antidepressant drugs, but in clinical practice such combinations are sometimes used.

\section{L-tryptophan}

There is evidence from controlled trials that the addition of L-tryptophan can improve the therapeutic effect of MAOI treatment. However, there are no controlled trials to indicate that L-tryptophan can reliably produce therapeutic benefit in patients who have failed to respond to MAOIs or TCAs. Nevertheless, the use of L-tryptophan has been recommended to supplement the 5-HT potentiating effects of lithium-MAOI and lithium-clomipramine combinations (Barker et al, 1987; Hale et al, 1987).

L-tryptophan has been associated with the development of the eosinophilia myalgic syndrome, a severe connective tissue disease that can have a fatal outcome. Subsequent studies have shown that eosinophilia myalgic syndrome was almost certainly caused by a contaminant that occurred in the production of L-tryptophan from a single manufacturing source (Slutsker et al, 1990). In the UK it remains possible to prescribe L-tryptophan, in combination with other antidepressant drugs, for patients with chronic treatment-resistant depression. It should be noted, however, that the combination of L-tryptophan with MAOIs can lead to 5-HT neurotoxicity, so caution is needed. In addition L-tryptophan given with SSRIs can also result in 5-HT toxicity, so this combination is not recommended (Sternbach, 1991).

\section{Combining TCAs and MAOIs}

The combination of TCAs and MAOIs has been in use since the 1960s when the efficacy of this regime was first strongly advocated. Although the combination of MAOIs and TCAs is reported to be hazardous, the risks of significant interaction can be minimised if reasonable precautions are taken. These include avoiding imipramine and 
clomipramine, and starting the drugs together at low dose or adding the MAOI cautiously to established TCA treatment (see Chalmers \& Cowen, 1990).

In patients not selected for treatment resistance the combination of MAOIs and TCAs does not appear to confer additional therapeutic benefit over either drug used alone. However, Sethna (1974) carried out an open study of MAOI-TCA treatment in 12 patients with depression who had failed to respond to either TCAs or MAOIs given separately (or electroconvulsive therapy (ECT) in 10 cases). At follow-up periods of 7-24 months, nine subjects were reported to be without significant depressive symptomatology. Most of these subjects had chronic non-melancholic depression with prominent anxiety symptoms.

In addition to these series, case reports continue to appear where it seems well documented that a patient has failed to respond to either a TCA or an MAOI given alone, but achieves a good clinical response when both drugs are used together (Tyrer \& Murphy, 1990). Therefore, although controlled evidence is lacking, it seems likely that individual patients with refractory depression are helped by MAOI-TCA combinations. Generally, the adverse effects of the combination are no worse than with either drug alone, although weight gain and postural hypotension may be more troublesome. Conversely, if an MAOI is given with a TCA such as amitriptyline or trimipramine, MAOI-induced insomnia may be prevented.

There is less information about the combination of other antidepressants with MAOIs. However, trazodone in doses of $50-150 \mathrm{mg}$ is fairly commonly used to treat MAOI-induced insomnia and is generally well-tolerated (Nierenberg \& Keck, 1989).

\section{Combining TCAs and SSRIs}

Some open case series have suggested that combining TCA and SSRI treatment may be helpful in refractory depression. For example, in a retrospective chart review, Weilburg et al (1989) reported a positive response (defined as improvement noted by both patient and clinician) in 22 of 25 out-patients with depression when fluoxetine was added to ongoing TCA treatment. This study does not clarify whether or not the improvement was due to the fluoxetine alone or the combination of fluoxetine with the TCA. However, in eight patients the therapeutic response was lost when the TCA was withdrawn, and restored was it was recommenced.

Subsequently, Weilburg et al (1991), in a similar study, found resolution of depression in 13 of 20 out-patients in whom nortriptyline or desipramine were added to ineffective fluoxetine treatment. One patient worsened with severe agitation. Finally, Seth et al (1992) reported remarkable improvement in eight elderly patients with chronic refractory depression who received an SSRI-TCA combination (usually sertraline and nortriptyline). Some patients received concomitant lithium treatment.

The only prospective study of TCA-SSRI treatment was carried out by Fava et al (1994) who treated 41 out-patients suffering with depression who had failed to achieve a $50 \%$ reduction in HAM-D scores in response to a standard dose of fluoxetine $(20 \mathrm{mg}$ daily). Subjects were randomly allocated to three different groups: (a) high-dose fluoxetine (40-60 mg daily); (b) the addition of desipramine (25-50 mg daily); or (c) lithium. Taking response as a final HAM-D score (at five weeks) of $<7$, the most effective treatment was high-dose fluoxetine ( $53 \%$ response rate) while lithium and desipramine augmentation appeared of similar efficacy (response rates of $29 \%$ and $25 \%$ respectively). However, the plasma levels of lithium obtained in this study were low and probably sub-therapeutic.

Taken together the controlled evidence for the efficacy of TCA-SSRI combinations is not compelling. In addition there are numerous case reports of adverse reactions with agitation and, rarely, seizures. These reactions are generally associated with marked elevations in plasma TCA levels because SSRIs are potent inhibitors of the cytochrome P450 system by which TCAs are metabolised. While some SSRIs, for example, citalopram and sertraline, may be less likely to produce this effect, the use of low doses of TCAs in conjunction with plasma monitoring is advisable if combination treatment is used (Taylor, 1995). Another option may be to use treatment with a single drug such as clomipramine or venlafaxine which produces potent $5-\mathrm{HT}$ and noradrenaline reuptake inhibition.

Another drug commonly used in combination with SSRIs is trazodone. Although there are some uncontrolled data suggesting that trazodone can augment the antidepressant effects of SSRIs (Weilburg et al, 1991), the usual reason for employing this combination is that the hypnotic effect of trazodone can amelioriate SSRI-induced sleep disturbance. Low doses of trazodone (50-150 mg) are usually sufficient and the combination is generally well tolerated, although there are rare reports of symptoms suggestive of serotonin toxicity.

\section{Pindolol}

Repeated administration of a number of antidepressant drugs, particularly SSRIs and MAOIs, desensitises inhibitory 5- $\mathrm{HT}_{1 \mathrm{a}}$ autoreceptors on 5$\mathrm{HT}$ cell bodies. It has been suggested that this effect contributes to the antidepressant effect of such drugs 
by freeing 5 -HT cell bodies from feedback control and thereby facilitating the release of 5-HT from nerve terminals (Blier \& de Montigny, 1994).

Based on this idea, Artigas et al (1994) proposed that the addition of a 5- $\mathrm{HT}_{1 \mathrm{a}}$ receptor antagonist to the medication of patients who had not responded to conventional antidepressants, particularly SSRIs, might produce a therapeutic effect. Because there are at present no selective $5-\mathrm{HT}_{1 \mathrm{a}}$ receptor antagonists available for clinical use, these authors employed pindolol, a $\beta$-adrenoceptor antagonist with 5-HT ${ }_{1 \mathrm{a}}$ receptor antagonist properties.

Pindolol (2.5 $\mathrm{mg}$ three times daily) was added to the drug treatment of seven patients with major depression who had been resistant to multiple medication trials. Five subjects were currently taking an SSRI (four paroxetine, one fluvoxamine) while one received imipramine and one phenelzine. All subjects showed a decrease in HAM-D score of at least $50 \%$ after a week of pindolol addition and in five, HAM-D scores were less than eight, indicating full remission (Artigaset al, 1994).

Subsequently, Blier \& Bergeron (1995) reported on the addition of pindolol to 18 patients with major depression who had failed to respond to treatment with an antidepressant together with the addition of another treatment, usually lithium. Pindolol ( $2.5 \mathrm{mg}$ three times daily) was added to paroxetine (eight subjects), sertraline (five subjects), fluoxetine (three subjects) and moclobemide (two subjects). Overall, pindolol produced a significant improvement in depressive symptoms after one week. By two weeks all the patients, except those on sertraline, had a HAM-D score of 10 or less showing a good clinical improvement. Interestingly, none of the patients treated with sertraline responded. It is not clear whether or not this may represent a chance finding. Despite the intriguing results from open studies a recent small controlled study of pindolol addition to ineffective SSRI treatment showed no benefit over placebo (Morenoet al, 1997).

Pindolol addition is generally well tolerated, although careful clinical screening is needed to exclude patients with asthma or cardiac conduction disturbances. In open studies pindolol addition has been associated with irritability and one patient developed mania.

\section{Electroconvulsive therapy}

Among the indications for ECT is that of failure to respond to adequate antidepressant drug treatment. Trials of ECT typically report high response rates (about $80 \%$ ), but patients who are unresponsive to drug treatment are not usually considered as a separate group.
Prudic et al (1990) studied the effect of previous antidepressant drug treatment on the response of 53 patients who received bilateral ECT. They found that among those who had received adequate pharmacotherapy (a TCA at a dose of at least $200 \mathrm{mg}$ daily for at least four weeks) the response rate to ECT (defined as a $60 \%$ reduction in HAM-D score) was $50 \%$. In contrast, the response rate of patients who had not received adequate drug treatment was significantly greater $(86 \%)$. The presence of medication resistance, therefore, decreases the likelihood that a patient will respond to ECT. Nevertheless, at least half of such subjects are likely to experience significant improvement.

Another point that needs to be considered is the outcome after ECT. Sackeim et al (1990) followed 58 patients who responded to ECT and found that oneyear post-treatment, $50 \%$ had relapsed. The relapse rate in patients who had received adequate drug treatment prior to ECT was significantly higher than in those who had not (64 v. 32\%). The relapse rate after ECT was only weakly influenced by whether or not patients received adequate antidepressant drug treatment post-ECT.

The practical point from this is that if patients have been unresponsive to a particular medication prior to ECT, continuing with this medication after successful ECT treatment may not provide effective prophylaxis. Therefore consideration should be given to using another class of antidepressant or perhaps lithium prophylaxis if this has not been tried previously. Development of appropriate continuation therapy after ECT is a research priority.

ECT can be used at any point in the treatment of resistant depression. Clinical features that will encourage earlier use include severe depression with high acute suicidal risk, depressive psychosis and pronounced psychomotor features, especially where fluid intake is compromised.

\section{Bipolar depression}

The management of bipolar depression is outside the scope of this article, but it is worth noting that it poses additional problems to those encountered in the treatment of resistant unipolar depression. Among these problems are the possibility of induction of mania or rapid cycling by antidepressant drugs. Where patients are having what appears to be a rapidly relapsing depressive illness it is important to establish whether or not the clinical picture is, in fact, rapid cycling with periods of mild hypomania interspersed with depression (Hurowitz \& Liebowitz, 1993). Recording of daily moods by the patient can be helpful in clarifying the diagnosis. If rapid cycling is confirmed, withdrawal 
of antidepressant treatment and the institution of mood stabilising medication can be a useful strategy.

In the treatment of bipolar illness the primary aim is that of mood stabilisation, and there is scope for the use of mood stabilising agents such as carbamazepine in depressive spells (Cowen, 1998). The clinical impression is that antidepressant drugs often seem somewhat less effective in bipolar depression, although bipolar depression with features of anergia and hypersomnia may respond better to MAOIs than to TCAs (Himmelhoch et al, 1991).

\section{Conclusion}

General and old age psychiatrists need to have confidence in their ability to manage the pharmacological aspects of treatment-resistant depression. It is important to retain the belief that ultimately the patient can recover, because it continues to be the case that even several years of severe depression can be followed by clinical remission (Mueller et al, 1996). At the same time it is necessary to recognise (and acknowledge to the patient) the limitations and discomforts of comtemporary drug treatments.

Patients are usually aware that miracles are not on offer but do appreciate a practitioner who systematically follows a carefully explained treatment plan. A stepwise approach organised along the lines suggested in this review is shown in

Box 3. Approach for pharmacological treatment of resistant depression

(a) Adjust treatment to maximum therapeutic dose dependent on tolerance (add antipsychotic drug if depressive psychosis)

(b) Switch antidepressant (e.g. TCA for SSRI and vice versa)

(c) Augment with lithium

(d) Augment with triiodothyronine

(e) MAOI (can continue with lithium)

(f) Other combinations (e.g. MAOI + TCA, lithium + MAOI + L - t r y p t o p h a n, lithium + clomipramine + L-tryptophan)

ECT can be used at any stage, depending on clinical features and the need for quick response
Box 3. It is always helpful to have in mind what the next step will be if the current treatment regime fails. Thase \& Rush (1997) warn that it is important for the clinician not to become demoralised or frustrated by a patient's lack of response and point out that even where promising pharmacological options appear to be limited, supportive psychological treatment has an important, life-sustaining function (Holmes, 1995).

\section{References}

American Psychiatric Association (1987) Diagnostic and Statistical Manual of Mental Disorders (3rd edn, revised)(DSM-III-R). Washington, DC: APA.

Anderson, I. (1997) Lessons to be learnt from meta-analyses of newer versus older antidepressants. Advances in Psychiatric Treatment, 3, 58-63.

Artigas, F., Perez, V. \& Alvarez, E. (1994) Pindolol induces a rapid improvement of depressed patients treated with serotonin reuptake inhibitors. Archives of General Psychiatry, 51, 248-251.

Barker, W. A., Scott, J. \& Eccelston, D. (1987) The Newcastle Chronic Depression Study: results of a treatment regime. International Clinical Psychopharmacology, 2, 261-272.

Blier, P. \& de Montigny, C. (1994) Current advances and trends in the treatment of depression. Trends in Pharmacological Sciences, 15, 220-226.

Blier, P. \& Bergeron, R. (1995) Effectiveness of pindolol with selective antidepressant drugs in the treatment of major depression. Journal of Clinical Psychopharmacology, 15, 217-222.

Chalmers, J. S. \& Cowen, P. J. (1990) Drug treatment of tricyclic resistant depression. International Review of Psychiatry, 2, 239-248.

Cowen, P. J. (1998) Treatments for resistant depression. In The Management of Depression (ed. S. Checkley), pp. 234251. London: Blackwell.

Fava, M., Rosenbaum, J. F., McGrath, P. J., et al (1994) Lithium and tricyclic augmentation of fluoxetine treatment for major depression: a double-blind controlled study. American Journal of Psychiatry, 151, 1372-1374.

Hamilton, M. (1967) Development of a rating scale for primary depressive illness. British Journal of Social and Clinical Psychology, 6, 278-296.

Hale, A. S., Procter, A. W. \& Bridges, P. K. (1987) Clomipramine, tryptophan and lithium in combination for resistant endogenous depression: seven case studies. British Journal of Psychiatry, 151, 213-217.

Himmelhoch, J. M., Thase, M. E., Mallinger, A. G., et al (1991) Tranylcypromine versus imipramine in anergic bipolar depression. American Journal of Psychiatry, 148, 910-916.

Holmes, J. (1995) Supportive psychotherapy. The search for positive meanings. British Journal of Psychiatry, 167, 439-455.

Hurowitz, G. I. \& Liebowitz, M. R. (1993) Antidepressantinduced rapid cycling: six case reports. Journal of Clinical Psychopharmacology, 13, 52-56.

Joffe, R. T., Singer, W., Levitt, A. J., et al (1993) A placebocontrolled comparison of lithium and triiodothyronine augmentation of tricyclic antidepressants in unipolar refractory depression. Archives of General Psychiatry, 50, 387-393.

Johnson, F. N. (1991) Lithium augmentation therapy for depression. Reviews in Contemporary Pharmacotherapy, 2, 3-52.

Katona, C. L. E., Abou-Saleh, M. T., Harrison, D. A., et al (1995) Placebo-controlled trial of lithium augmentation of fluoxetine and lofepramine. British Journal of Psychiatry, $166,80-86$ 
Kupfer, D.J. \& Frank, E. (1996) Maintenance therapy for chronic depression. Archives General Psychiatry, 53, 775-776.

Lindberg, G., Bingefors, K., Ranstam, J., et al (1998) Use of calcium channel blockers and risk of suicide: ecological findings confirmed in population based cohort study. British Medical Journal, 316, 741-745.

Moreno, F. A., Gelenberg, A. J., Bachar, K., et al (1997) Pindolol augmentation of treatment-resistant depressed patients. Journal of Clinical Psychiatry, 58, 437-439.

Mueller, T. J., Keller, M. B., Leon, A. C., et al (1996) Recovery after 5 years of unremitting major depressive disorder. Archives General Psychiatry, 53, 794-799.

Muller-Siecheneder, F., Muller, M. J., Hillert, A., et al (1998) Risperidone versus haloperidol and amitriptyline in the treatment of patients with a combined psychotic and depressive syndrome. Journal of Clinical Psychopharmacology, 18, 111-120.

Nierenberg, A. A. \& Keck, P. E. (1989) Management of monoamine oxidase inhibitor-associated insomnia with trazodone. Journal of Clinical Psychopharmacology, 9, 45-54.

Nolen, W. A., Van de Putte, J. J., Dijken, W. A., et al (1988) Treatment strategy in depression. II. MAO inhibitors in depression resistant tricyclic antidepressants: two controlled cross-over studies with tranylcypromine versus 1-5-hydroxytryptophan and nomifensine. Acta Psychiatrica Scandinavica, 78, 676-683.

Preskorn, S. H. (1993) Pharmacokinetics of antidepressants: Why and how are they relevant to treatment? Journal of Clinical Psychiatry, 54 (suppl. 9), 14-34.

Price, L. H., Charney, D. S. \& Heninger, G. R. (1985) Efficacy of lithium-tranylcypromine treatment in refractory depression. American Journal of Psychiatry, 142, 619-623.

Prudic, J., Sackeim, H. A. \& Devanand, D. P. (1990) Medication resistance and clinical response to electroconvulsive therapy. Psychiatry Research, 31, 287-296.

Quitkin, F. M., McGrath, P. J., Stewart, J. W., et al (1989) Phenelzine and imipramine in mood reactive depressives. Archives of General Psychiatry, 46, 787-793.

Rothschild, A. J., Samson, J. A., Bessette, M. P., et al (1993) Efficacy of the combination of fluoxetine and perphenazine in the treatment of psychotic depression. Journal of Clinical Psychiatry, 54, 338-342.

Sackeim, H. A., Prudic, J., Devenand, D. P., et al (1990) The impact of medication resistance and continuation of pharmacotherapy on relapse following response to electroconvulsive therapy in major depression. Journal of Clinical Psychopharmacology, 10, 96-104.

Seth, R., Jennings, A. L., Bindman, J., et al (1992) Combination treatment with noradrenaline and serotonin reuptake inhibitors in resistant depression. British Journal of Psychiatry, 161, 562-565.

Sethna, E. R. (1974) A study of refractory cases of depressive illnesses and the response to combined antidepressant treatment. British Journal of Psychiatry, 124, 265-272.

Slutsker, L., Hoesly, F. C., Miller, L., et al (1990) Eosinophiliamyalgia syndrome associated with exposure to tryptophan from a single manufacturer. Journal of the American Medical Association, 264, 213-217.

Spiker, D. G., Weiss, J. C. \& Dealy, R. S. (1985) The pharmacological treatment of delusional depression. American Journal of Psychiatry, 142, 430-436.

Stabl, M., Kasas, A., Blajev, B., et al (1995) A double-blind comparison of moclobemide and thioridazine versus moclobemide and placebo in the treatment of refractory severe depression. Journal of Clinical Psychopharmacology, 15 (suppl. 2), 41S-45S.

Sternbach, H. (1991) The serotonin syndrome. American Journal of Psychiatry, 148, 705-713.

Taylor, D. (1995) Selective serotonin reuptake inhibitors and tricyclic antidepressants in combination: interactions and therapeutic uses. British Journal of Psychiatry, 167, 575-580.

Thase, M. E. \& Rush, A. J. (1997) When at first you don't succeed: sequential strategies for antidepressant nonresponders. Journal of Clinical Psychiatry, 58 (suppl. 13), 23-29.
Tyrer, P. \& Murphy, S. (1990) Efficacy of combined antidepressant therapy in resistant neurotic disorder. British Journal of Psychiatry, 156, 115-118.

Weilburg, J. B., Rosenbaum, J. F., Biderman, J., et al (1989) Fluoxetine added to non-MAOI antidepressants converts non-responders to responders: a preliminary report. Journal of Clinical Psychiatry, 50, 447-449.

-, - Meltzer-Brody, S., et al (1991) Tricyclic augmentation of fluoxetine. Annals of Clinical Psychiatry, 3, 209-213.

Young, A. H. \& Cowen, P. J. (1994) Antidepressant drugs. In Side-Effects of Drugs Annual, Vol. 17 (eds J. K. Aronson \& C. J. Van Boxtel), pp. 16-25. Amsterdam: Elsevier.

\section{Multiple choice questions}

1. In the treatment of resistant depression, monoamine oxidase inhibitors:

a are not effective in patients who have failed to respond to ECT

b may be effective in patients with hypersomnia and hyperphagia

c can cause postural hypotension at higher doses

d should not be combined with lithium.

2. Controlled trials have shown that the following treatments can augment tricyclic antidepressant treatment in unresponsive patients:
a lithium
b thyroxine
c pindolol
d triiodothyronine.

3. Lithium augmentation of ineffective antidepressant treatment:

a does not work in unipolar depression

b is contraindicated with SSRIs

c requires a plasma concentration of $0.5-$ $0.8 \mathrm{mmol} / \mathrm{l}$

$\mathrm{d}$ works within 48 hours in most patients.

4. The following combinations can cause severe drug interactions:

a SSRIs and tricyclic antidepressants

b SSRIs and tryptophan

c amitryptyline and triiodothyronine

d lithium and triiodothyronine.

\section{MCQ answers}

1

a $\mathrm{F}$

b $T$

2

c $\mathrm{T}$

a $\mathrm{T}$

b $\mathrm{F}$

d $F$

c $\mathrm{F}$

d $\mathrm{T}$

3

4

a $\mathrm{F}$

a $\mathrm{T}$

b $\mathbf{F}$

b $T$

c $\mathrm{T}$

c $\mathrm{F}$

d $\mathbf{F}$ 